\title{
Accurate predictions for the scale-dependent galaxy bias from primordial non-Gaussianity
}

\author{
Vincent Desjacques, ${ }^{1}$ Donghui Jeong, ${ }^{2}$ and Fabian Schmidt ${ }^{2}$ \\ ${ }^{1}$ Institute for Theoretical Physics, University of Zürich, Winterthurerstrasse 190, CH-8057 Zürich, Switzerland \\ ${ }^{2}$ California Institute of Technology, Mail Code 350-17, Pasadena, California, 91125, USA
}

(Received 20 May 2011; published 6 September 2011)

\begin{abstract}
The large-scale clustering of galaxies can serve as a probe of primordial non-Gaussianity in the Universe competitive with the anisotropies of the cosmic microwave background. Here, we present improved theoretical predictions which include an important, previously overlooked correction to the bias. We demonstrate that the new predictions are able to reproduce the results of N-body simulations, explaining the significant departures seen from previous theoretical results. These refined predictions open the way to accurate constraints on primordial physics with large-scale structure surveys.
\end{abstract}

DOI: 10.1103/PhysRevD.84.061301

PACS numbers: 98.80.Es, 95.80.+p, 98.65.Cw, 98.65.Dx

Measurements of the temperature and polarization anisotropies of the cosmic microwave background (CMB) radiation strongly support inflation as the mechanism generating the initial curvature perturbations [1], here parametrized by the Bardeen potential perturbations $\phi=\Phi_{H}[2]$. While the simplest, single-field slow-roll models predict nearly Gaussian fluctuations, many other inflationary scenarios yield a potentially detectable level of primordial non-Gaussianity, which can generally be described in terms of the higher-order $(N \geq 3)$ correlation functions of $\phi$ in Fourier space, $\xi_{\phi}^{(N)}$. Because different seeding mechanisms of the initial curvature perturbations leave distinct signatures in the shape and amplitude of these higher-order correlation functions, a precise measurement of non-Gaussianity would strongly constrain the physics of inflation.

As the primordial curvature perturbations eventually grow into the large-scale structure observed today, their higher-order correlation functions can be determined through a measurement of the clustering of dark matter halos [3] which host the galaxies. For example, primordial non-Gaussianity of the local $f_{\mathrm{NL}} \phi^{2}$-type induces a strongly scale-dependent bias $\Delta \mathrm{b}_{\mathrm{I}}(k, z)$ in the clustering of halos [4]. The power spectrum $P_{h}$ of halos of mass $M$ at redshift $z$ is related to the matter power spectrum $P_{m}$ through

$$
\begin{aligned}
P_{h}(k, M, z) & =\left[\mathrm{b}_{\mathrm{I}}^{\mathrm{E}}(k, M, z)\right]^{2} P_{m}(k, z) \\
& =\left[b_{1}^{\mathrm{E}}(M, z)+\Delta \mathrm{b}_{\mathrm{I}}(k, M, z)\right]^{2} P_{m}(k, z) \\
\Delta \mathrm{b}_{\mathrm{I}}(k, M, z) & =\left(b_{1}^{\mathrm{E}}(M, z)-1\right) \frac{2 f_{\mathrm{NL}} \delta_{c}}{\mathcal{M}(k, z)}
\end{aligned}
$$

Here, $b_{1}^{\mathrm{E}}(M, z)$ is the linear, Gaussian (Eulerian) bias of halos ( $b_{1} \equiv b_{1}^{\mathrm{E}}-1$ is the corresponding Lagrangian bias), $\delta_{c} \simeq 1.69$ is the critical density for spherical collapse, and the function $\mathcal{M}(k, z) \equiv 2 k^{2} T(k) D(z) /\left(3 \Omega_{m} H_{0}^{2}\right)$ depends on the linear growth factor $D(z)$, the matter transfer function $T(k)$, the present-day matter density parameter $\Omega_{m}$ and the Hubble constant $H_{0}$. In what follows, we shall omit the mass and redshift dependence for conciseness.

The scale-dependent non-Gaussian (NG) bias expression [Eq. (2)] may be extended (for $N=3$ ) using a peakbackground split (PBS) argument [5]. Alternatively, Eq. (2) may be generalized to any model of primordial NG by computing the correlation function of high threshold regions [6]. Even though the result is strictly valid in the high-peak limit only, i.e. $\nu \equiv \delta_{c} / \sigma_{0 s} \gg 1$ where $\sigma_{0 s}$ is the variance of the density field smoothed on scale $R_{s}(M)=(3 M / 4 \pi \bar{\rho})^{1 / 3}$, it is usually extended to arbitrary peak heights upon identifying $\nu / \sigma_{0 s}$ with $b_{1}$. For a given arbitrary $N$, this gives

$$
\Delta \mathrm{b}_{\mathrm{I}}^{(\mathrm{hp})}(k)=\frac{4 b_{1} \delta_{c}}{(N-1) !}\left(\frac{\nu}{\sigma_{0 s}}\right)^{N-3} \frac{\mathcal{F}_{s}^{(N)}(k)}{\mathcal{M}_{s}(k)} .
$$

We have defined $\mathcal{M}_{s}(k)=\mathcal{M}(k) W_{R_{s}}(k)$, where $W_{R_{s}}(k)$ is the Fourier transform of the spherical tophat filter with radius $R_{s}$. Furthermore, we have introduced the shape factor

$$
\begin{aligned}
\mathcal{F}_{s}^{(N)}(k)= & \frac{1}{4 \sigma_{0 s}^{2} P_{\phi}(k)}\left[\prod_{i=1}^{N-2} \int \frac{d^{3} k_{i}}{(2 \pi)^{3}} \mathcal{M}_{s}\left(k_{i}\right)\right] \\
& \times \mathcal{M}_{s}(q) \xi_{\phi}^{(N)}\left(\boldsymbol{k}_{1}, \cdots, \boldsymbol{k}_{N-2}, \boldsymbol{q}, \boldsymbol{k}\right),
\end{aligned}
$$

with $\boldsymbol{q} \equiv-\boldsymbol{k}_{1}-\cdots-\boldsymbol{k}_{N-2}-\boldsymbol{k}$, and $P_{\phi}(k)$ being the power spectrum of the Bardeen potential $\phi$. For the constant $f_{\mathrm{NL}} \phi^{2}$ model, $\mathcal{F}_{s}^{(3)}(k) / f_{\mathrm{NL}}$ is equivalent to the function $\mathcal{F}_{R}(k)$ defined in [6]. As $\mathcal{F}_{s}^{(3)}(k) \rightarrow f_{\mathrm{NL}}$ on large scales, Eq. (3) reproduces Eq. (2) in the limit $k \rightarrow 0$. Since, in the limit $\nu \gg 1$, both thresholding and PBS approaches yield Eq. (3), we call it the high-peak result.

Measurements of the clustering of dark matter halos in non-Gaussian N-body simulations have confirmed the validity of Eq. (1). The $k$-dependent NG bias currently yields constraints on the nonlinear parameter $f_{\mathrm{NL}}$ competitive with CMB bispectrum measurements [4]. For other models of primordial non-Gaussianity however, comparisons 
between the high-peak expectation [Eq. (3)] and the simulated NG bias have revealed large discrepancies in the magnitude of $\Delta b_{\mathrm{I}}[7-9]$.

In [10], we calculate the scale-dependent NG bias using two different formulations of the peak-background split. The first generalizes the approach adopted in [4,5]; the second utilizes conditional mass functions. Both methods arrive at the same expression on large scales and thus validate the robustness of the result. Let us briefly review the second approach, which is inspired by a derivation of the Gaussian peak bias factors [11]. The conditional mass function $\bar{n}\left(M, R_{s} \mid \delta_{l}, R_{l}\right)$ gives the mean number density of halos of mass $M$ and radius $R_{S}$ inside a large region of size $R_{l} \gg R_{s}$ and overdensity $\delta_{l}$. The conditional mass function encodes information on the halo bias parameters $[12,13]$. On taking the limit $R_{l} \rightarrow \infty$ and expanding the conditional overabundance of halos,

$$
\left\langle\delta_{h} \mid \delta_{l}\right\rangle=\frac{\bar{n}\left(M, R_{s} \mid \delta_{l}, R_{l}\right)}{\bar{n}\left(M, R_{S}\right)}-1,
$$

in powers of the large-scale perturbation $\delta_{l}$, we are able to read off the NG bias factors. In particular, the scaledependent $N G$ contribution to $b_{\mathrm{I}}^{\mathrm{E}}$ is at leading order

$$
\begin{aligned}
\Delta \mathrm{b}_{\mathrm{I}}^{(\mathrm{pbs})}(k) & =\frac{4}{(N-1) !} \frac{\mathcal{F}_{s}^{(N)}(k)}{\mathcal{M}_{s}(k)} \epsilon_{s}(k) \\
\epsilon_{s}(k) & \equiv b_{N-2} \delta_{c}+b_{N-3}\left(3-N+\frac{d \ln \mathcal{F}_{s}^{(N)}(k)}{d \ln \sigma_{0 s}}\right),
\end{aligned}
$$

where $b_{N}$ is the $N$-th order, Gaussian Lagrangian bias (with $\left.b_{0} \equiv 1\right)$. The first term $\left(b_{N-2} \delta_{c}\right)$ reduces to Eq. (3) for high mass halos since $b_{N} \simeq\left(\nu / \sigma_{0 s}\right)^{N}$ when $\nu \gg 1$. Therefore, it exactly reproduces the previous results of $[4,5,7,8]$. The second term, which is derived for the first time in [10], only vanishes in the local, quadratic $f_{\mathrm{NL}}$ model and on large scales. Although it becomes subdominant in the high-peak limit $\left(b_{N-2} / b_{N-3} \gg 1\right)$, it is significant for most relevant peak heights.

To decipher the origin of this new term, it is useful to recall the basic mechanism by which primordial nonGaussianity affects halo clustering. In the presence of a primordial bispectrum, the small-scale density field is modulated by a long-wavelength $(k \lesssim 0.01 \mathrm{~h} / \mathrm{Mpc})$ perturbation of the potential $\phi$ via $[4,5]$

$$
\hat{\sigma}_{0 s}^{2}(k)=\sigma_{0 s}^{2}\left(1+4 \mathcal{F}_{s}^{(3)}(k) \phi_{l}(\mathbf{k})\right) .
$$

Thus, instead of being a constant throughout space, $\hat{\sigma}_{0 s}^{2}$ and hence $\nu$ vary from point to point. Since the local abundance of halos depends on the amplitude of small-scale density fluctuations (it is commonly considered to be proportional to some multiplicity function $f(\nu)$ ), the change in $\hat{\sigma}_{0 s}$ [Eq. (7)] contributes to the variation in the overdensity $\delta_{h}$ of halos. Most importantly, this modulation of $\hat{\sigma}_{0 s}$ induces a dependence of $\delta_{h}$ on $\phi$, rather than $\delta_{m}$. This explains why the scale-dependent NG bias generally increases towards larger scales (but note the additional $k$ dependence brought by $\mathcal{F}_{s}^{(3)}(k)$ ). As shown in [10], similar arguments can be made for a generic primordial $N$-point function. In this case, long-wavelength potential perturbations locally induce a reduced moment of order $(N-1)$ in the small-scale density field $\delta_{s}$, which is given by

$$
\hat{\mathcal{S}}_{s}^{(N-1)}(k) \equiv \frac{\left\langle\delta_{s}^{N-1}\right\rangle}{\sigma_{0 s}^{N-1}}=4 \sigma_{0 s}^{3-N} \mathcal{F}_{s}^{(N)}(k) \phi_{l}(\mathbf{k}) .
$$

For example, a primordial trispectrum generates a local skewness. Therefore, we can think of the effect of a primordial $\mathrm{N}$-point function as locally rescaling the local significance according to [10]

$$
\nu \rightarrow \hat{\nu} \equiv \nu\left(1-\frac{4}{(N-1) !} b_{N-3} \mathcal{F}_{s}^{(N)}(k) \phi_{l}(\mathbf{k})\right) .
$$

Clearly, the modulation of the variance, skewness etc. of the small-scale density field not only changes the significance $\nu$ that corresponds to a mass $M$, but also affects the significance interval $d \nu$ which a fixed mass bin $d M$ is mapped to. Hence, long-wavelength perturbations of the Jacobian $d \ln \hat{\nu} / d \ln M$ generate a dependence of the nonGaussian halo bias on the derivative of the shape factor $\mathcal{F}_{s}^{(3)}$ w.r.t. $R_{s}$. This term must be present because halos in $\mathrm{N}$-body simulations and, to a lesser extent, in galaxy surveys are identified by mass. In contrast, in the thresholding approach the two-point correlation function of halos above a mass threshold $M$ is associated with that of Lagrangian regions above a threshold $\delta_{c}$ in the linear density field smoothed on a fixed scale $R_{s}(M)$. This is the reason why this approach cannot recover the second term in the expression of $\boldsymbol{\epsilon}_{s}(k)$ [Eq. (6)].

In the remainder of this Letter, we shall compare the prediction Eq. (6) to simulation results in the large-scale limit $k \rightarrow 0$. In this regard, it is convenient to express both the predicted and simulated scale-dependent NG bias in units of the high-peak expectation Eq. (3), i.e. we plot $\Delta b_{\mathrm{I}} / \Delta \mathrm{b}_{\mathrm{I}}^{\mathrm{hp}}$. Our prediction for this ratio is

$$
\frac{\Delta \mathrm{b}_{\mathrm{I}}^{(\mathrm{pbs})}(k)}{\Delta \mathrm{b}_{\mathrm{I}}^{(\mathrm{hp})}(k)}=\left(\frac{\nu}{\sigma_{0 s}}\right)^{3-N} \frac{\epsilon_{s}(k)}{b_{1} \delta_{c}}
$$

for a given primordial $N$-point correlation function. Note that, in the models we consider below, $\epsilon_{s}$ depends very weakly on $k$ for wave numbers $k \leqq 0.01 h / \mathrm{Mpc}$. Hence, Eq. (10) predicts a mass-dependent correction to the amplitude of the scale-dependent NG bias on large scales.

We consider the following three models beyond the scale-independent local $f_{\mathrm{NL}}$ for which simulations have been performed: 
(i) scale-dependent $f_{\mathrm{NL}}[8]$

$$
\xi_{\phi}^{(3)}\left(\mathbf{k}_{1}, \mathbf{k}_{2}, \mathbf{k}_{3}\right)=f_{\mathrm{NL}}\left(k_{p}\right)\left(\frac{k_{1}}{k_{p}}\right)^{n_{f}} P_{2} P_{3}+(5 \text { perm. })
$$

(ii) orthogonal bispectrum [9]

$$
\begin{aligned}
\xi_{\phi}^{(3)}\left(\mathbf{k}_{1}, \mathbf{k}_{2}, \mathbf{k}_{3}\right)= & 6 f_{\mathrm{NL}}\left[P_{1} P_{2}+(2 \text { perm. })\right. \\
& +3\left(P_{1} P_{2} P_{3}\right)^{2 / 3} \\
& \left.-\left(P_{1}^{1 / 3} P_{2}^{2 / 3} P_{3}+(5 \text { perm. })\right)\right]
\end{aligned}
$$

(iii) local $g_{\mathrm{NL}}$ model [7]

$$
\xi_{\phi}^{(4)}\left(\mathbf{k}_{1}, \mathbf{k}_{2}, \mathbf{k}_{3}, \mathbf{k}_{4}\right)=6 g_{\mathrm{NL}}\left[P_{1} P_{2} P_{3}+(3 \text { perm. })\right],
$$

where $P_{i} \equiv P_{\phi}\left(k_{i}\right)$. The shape factor for each of these models on large scales, respectively, asymptotes to

$$
\begin{gathered}
\mathcal{F}_{s}^{(3)}(k)=f_{\mathrm{NL}}\left(k_{p}\right) k_{p}^{-n_{f}}\left(\frac{\sigma_{\alpha s}}{\sigma_{0 s}}\right)^{2}, \quad \alpha=n_{f} / 2 \\
\mathcal{F}_{s}^{(3)}(k)=-3 f_{\mathrm{NL}}\left(\frac{\sigma_{\alpha s}}{\sigma_{0 s}}\right)^{2} k^{-2 \alpha}, \quad \alpha=\left(n_{s}-4\right) / 6 \\
\mathcal{F}_{s}^{(4)}(k)=\frac{3}{4} g_{\mathrm{NL}} \sigma_{0 s}^{2} S_{s, \mathrm{loc}}^{(3)},
\end{gathered}
$$

where the spectral moments are defined as

$$
\sigma_{n s}^{2} \equiv \int \frac{d^{3} k}{(2 \pi)^{3}} k^{2 n} P_{\phi}(k) \mathcal{M}_{s}^{2}(k) .
$$

In Eq. (16), $S_{s, \text { loc }}^{(3)}$ is the skewness of the density field smoothed on scale $R_{s}$ in a local quadratic model with $f_{\mathrm{NL}}=1$. Using these expressions, it is straightforward to evaluate the ratio Eq. (10).

Figure 1 shows the simulations results of [8] for the local, scale-dependent $f_{\mathrm{NL}}$-type model [Eq. (11)] relative to the high-peak prediction, as a function of the Gaussian halo bias $b_{1}^{\mathrm{E}}$. The values of $k_{p}=0.04 \mathrm{Mpc}^{-1}$ and $f_{\mathrm{NL}}\left(k_{p}\right)=630$ are fixed, while the spectral index is $n_{f}= \pm 0.6$. Clearly, the data points are inconsistent with the high-peak prediction in the range $b_{1}^{\mathrm{E}} \lesssim 4$. The improved theoretical prediction presented in this paper explains the observed deviation of the high-peak prediction for the $z=0$ case (filled symbols, solid lines) and for the $z=1$ case and negative $n_{f}$ (open symbols, dashed lines). The only exception is for $n_{f}=0.6$ at $z=1$, where the $\mathrm{N}$-body data appears consistent with the high-peak prediction. This case deserves further investigation. Note however that the simulation measurements are correlated, since

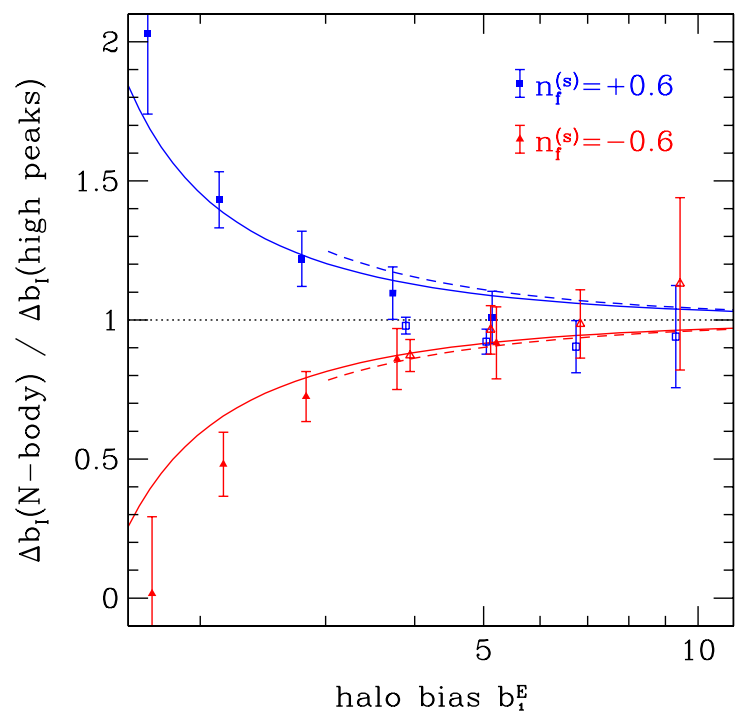

FIG. 1 (color online). Ratio of the large-scale NG halo bias measured in simulations with non-Gaussianity of the scaledependent local type $\left(n_{f}= \pm 0.6\right)$ [8], to that predicted by the high-peak approximation. Filled and open symbols show the simulation results, whereas the solid and dashed curves indicate the new theoretical expectation [Eq. (10)] at $z=0$ and 1, respectively.

they were estimated from a common simulation volume. Hence, the significance of this departure is not straightforward to estimate.

The results from simulations of the orthogonal bispectrum [Eq. (12)] are shown in Fig. 2 [9]. Symbols represent

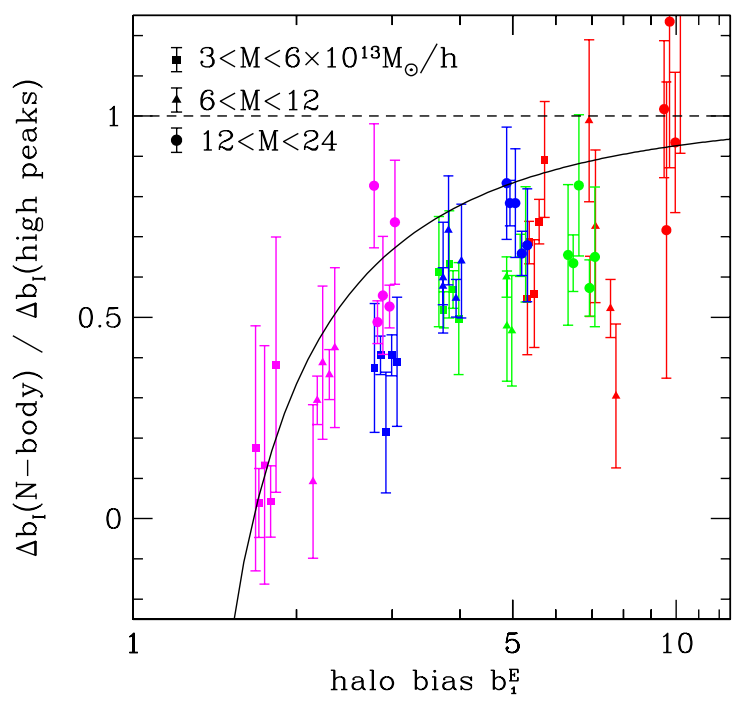

FIG. 2 (color online). Same as Fig. 1 but for the orthogonal bispectrum shape [9]. Different symbols correspond to various halo mass bins as indicated in the figure; colors indicate the redshifts $z=0$ (magenta), 0.67 (blue), 1 (green) and 1.5 (red). The solid curve shows the new theoretical prediction assuming a halo mass $M=5 \times 10^{13} M_{\odot} / h$. 


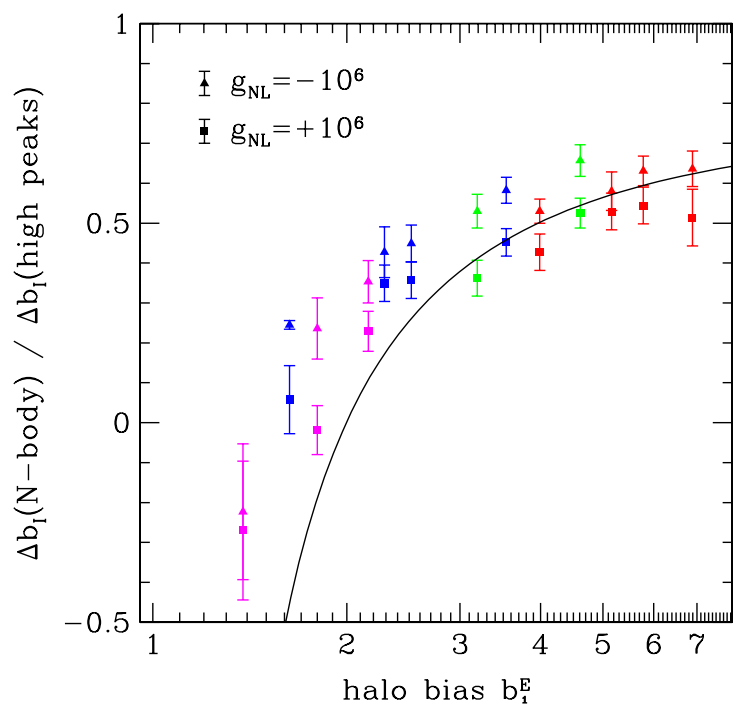

FIG. 3 (color online). Same as Fig. 1 but for the local cubic model. Triangle and square symbols indicate results for $g_{\mathrm{NL}}=-10^{6}$ and $10^{6}$, respectively. Different colors show different redshifts spanning the range $0<z<2$.

the measured NG halo bias from simulations relative to the high-peak prediction from three realizations with $f_{\mathrm{NL}}=-250$ and two with $f_{\mathrm{NL}}=-1000$. The results were averaged over wave numbers with $k<0.1 h \mathrm{Mpc}^{-1}$. We show our prediction (Eq. (10) with $N=3$ together with Eq. (15)) as the solid curve, for a median halo mass $M=5 \times 10^{13} M_{\odot} / h$. Again, the improved theory is consistent with the N-body data, which convincingly shows a strong suppression for $b_{1}^{\mathrm{E}} \lessgtr 2$. While the error bars are significant, the strong mass- and redshift-dependence predicted by Eq. (6) appears to be supported by the data.

Finally, Fig. 3 shows the ratio of the simulated largescale bias of the local $g_{\mathrm{NL}}$ model [Eq. (13)] to the highpeak prediction. The simulations used values of $g_{\mathrm{NL}}=$ $\pm 10^{6}$ [7]. Error bars indicate the scatter among 5 realizations. Clearly, the measured values of $\Delta b_{I}$ lie far below the high-peak prediction. While they increase monotonically with $b_{1}^{\mathrm{E}}, \Delta \mathrm{b}_{\mathrm{I}} / \Delta \mathrm{b}_{\mathrm{I}}^{(\mathrm{hp})}$ never reaches unity even for the most biased samples. The solid curve represents the improved theoretical prediction, Eq. (10) with the shape factor
Eq. (16). For this Figure, we have computed the Gaussian peak-background split biases $b_{1}$ and $b_{2}$ from a Sheth-Tormen mass function [13]. The prediction matches the measurements well, though it somewhat underestimates $\Delta \mathrm{b}_{\mathrm{I}}$ for $b_{1}^{\mathrm{E}} \lesssim 2$; an improved match might be achieved by measuring the $b_{2}$ of halos directly from simulations, rather than using the fitting function of [13]. Note also that the measurements are noticeably larger for $g_{\mathrm{NL}}=-10^{6}$, suggesting that second order contributions in $g_{\mathrm{NL}}$ are important. This is presumably due to the fact that the Gaussian bias factors $b_{1}$ and $b_{2}$ receive scaleindependent NG corrections which depend on the nonlinear parameter $g_{\mathrm{NL}}$.

In summary, we have presented a new formula for the NG bias based on an improved PBS argument [10]. We have obtained an additional term which can be interpreted as the effect of non-Gaussianity on the mapping between the significance $\nu$ and the mass of halos $M$. This new term vanishes only for the local quadratic model with constant $f_{\mathrm{NL}}$, and is significant for all other models considered here. We compare our theoretical predictions to the scaledependent bias measured from NG N-body simulations, and show that it is in good overall agreement with the measurements, in contrast to the existing formulae based on the statistics of high peaks. The improved theoretical predictions presented here will enable accurate upper limits on, or measurements of, general shapes of nonGaussianity. In particular, our results are of relevance to the recent measurements of [14], who employed the highpeak prediction, and are expected to increase their upper limits by factors of order unity. We expect similar massdependent corrections to the quadratic and higher-order bias factors which should significantly affect the halo bispectrum [10]. Accounting for all these corrections will be essential to obtain accurate constraints on primordial non-Gaussianity from large-scale structure.

We would like to thank S. Shandera and C. Wagner for providing the data shown in Fig. 1 and Fig. 2, respectively. D. J. and F. S. are supported by the Gordon and Betty Moore Foundation at Caltech. V. D. is supported by the Swiss National Foundation under Contract Nos. 200021116696/1 and FK UZH 57184001.
[1] E. Komatsu et al., Astrophys. J. Suppl. Ser. 192, 18 (2011).

[2] J. M. Bardeen, Phys. Rev. D 22, 1882 (1980).

[3] V. Desjacques and U. Seljak, Classical Quantum Gravity 27, 124011 (2010).

[4] N. Dalal, O. Doré, D. Huterer, and A. Shirokov, Phys. Rev. D 77, 123514 (2008); A. Slosar, C. Hirata, U. Seljak, S.
Ho, and N. Padmanabhan, J. Cosmol. Astropart. Phys. 8 (2008) 031.

[5] F. Schmidt and M. Kamionkowski, Phys. Rev. D 82, 103002 (2010).

[6] S. Matarrese and L. Verde, Astrophys. J. Lett. 677, L77 (2008); L. Verde and S. Matarrese, Astrophys. J. Lett. 706, L91 (2009); 
[7] V. Desjacques and U. Seljak, Phys. Rev. D 81, 023006 (2010).

[8] S. Shandera, N. Dalal, and D. Huterer, J. Cosmol. Astropart. Phys. 03 (2011) 017.

[9] C. Wagner and L. Verde, arXiv:1102.3229.

[10] V. Desjacques, D. Jeong, and F. Schmidt, arXiv:1105.3628 [Phys. Rev. D (to be published].
[11] V. Desjacques, M. Crocce, R. Scoccimarro, and R. K. Sheth, Phys. Rev. D 82, 103529 (2010).

[12] H. J. Mo and S. D. M. White, Mon. Not. R. Astron. Soc. 282, 347 (1996).

[13] R. K. Sheth and G. Tormen, Mon. Not. R. Astron. Soc. 308, 119 (1999).

[14] J.-Q. Xia et al., arXiv:1104.5015. 